\title{
Impedance Interaction Modeling and Analysis for Bidirectional Cascaded Converters
}

\author{
Yanjun Tian ${ }^{1}$, Fujin Deng ${ }^{1}$, Zhe Chen $^{1}$, Xiaofeng Sun ${ }^{2}$, Yanting $\mathrm{Hu}^{3}$ \\ ${ }^{1}$ Aalborg University, Denmark \\ ${ }^{2}$ Yanshan University, China \\ ${ }^{3}$ University of Chester, United Kingdom
}

\begin{abstract}
For the cascaded converter system, the output impedance of source converter interacts with the input impedance of load converter, and the interaction may cause the system instability. In bidirectional applications, when the power flow is reversed, the impedance interaction also varies, which brings more uncertainty to the system stability. An investigation is performed here for showing that the forward and reverse interactions are prominently different in terms of dynamics and stability even though the cascaded converter control remains unchanged. An important guideline has been drawn for the control of the cascaded converter. That is when voltage mode converter working as the load converter; the constant power mode converter as the source converter, the system is more stable. The concluded findings have been verified by simulation and experimental results.
\end{abstract}

Index Terms-- Cascaded converter, impedance interaction, bidirectional power flow, system stability.

\section{INTRODUCTION}

Cascaded converters are widely used in conventional power systems [1,2] and renewable distributed power systems $[3,4]$, because they can increase the system flexibility and compatibility. The topology of cascaded dual-active-bridges (DAB) with inverter is popular among researches $[5,6]$, so this paper discusses the impedance interaction based on this topology, as shown in Fig. 1.

For the cascaded system, the stability not only relies on the well designing of individual converter, but also the impedance interaction between the cascaded subconverters. The impedance interaction was analyzed by Middlebrook in 1979 [7], which showed that if the source converter output impedance is much less than the load converter input impedance, the stability of the cascaded system can be guaranteed, called Middlebrook criterion. After that several other criterions were developed in the past few years $[8,9]$.

In bidirectional application, the impedance interaction also becomes bidirectional. When power flow changes direction, previous source converter and load converter exchanges position with each other, which varies the impedance interaction. Conventional solutions are to switch the operation mode of sub-converter to adapt the changing of power flow direction [10,11], and normally they use the source converter to control DC-link voltage and load converter to control the load power, but there are stability issues in the constant power load [12], and they did not analysis the bidirectional impedance interaction, nor neither show the stability difference.

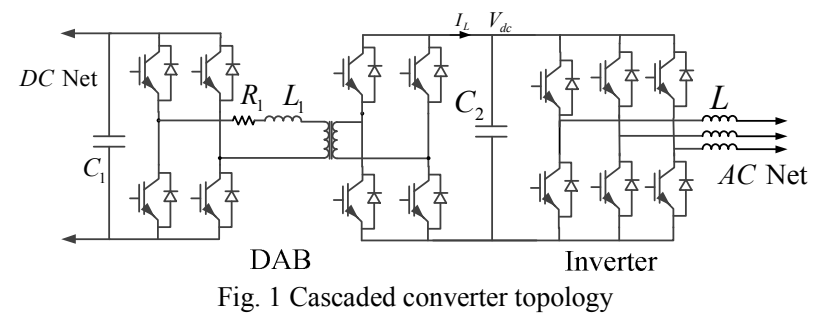

In this paper, the bidirectional impedance interaction has been modelled and analyzed. The analysis shows that the impedance interaction is different between forward power flow and reversed power flow, which can be concluded that when the voltage mode converter works as the load and constant power converter works as the source, the system is more stable.

\section{IMPEDANCE INTERACTION}

Fig. 2 shows the schematic of a cascaded system with two sub-converters in series.

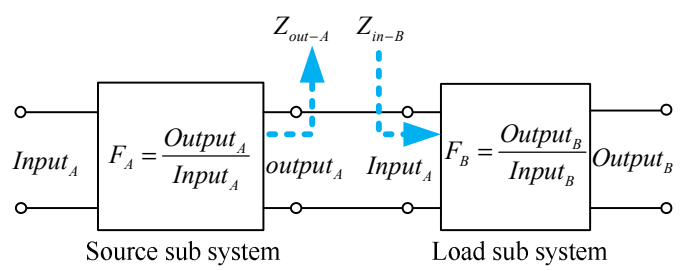

Fig. 2 Cascaded converter with impedance consideration

The overall cascade input to output transfer function $F$ can be expressed as [13]:

$$
F=F_{A} F_{B} \frac{1}{1+T_{m}}
$$

Where $F_{A}$ and $F_{B}$ are the individual transfer function, as shown in Fig. 2; $\frac{1}{1+T_{m}}$ is the impedance interaction. According to the control theory [14], $T_{m}$ can be recognized as the open loop transfer function of the impedance interaction, and it is called the minor loop gain [15]. Consequently, the stability of cascaded system will not only rely on individual well designing of the subconverters, but also the impedance interaction. If $T_{m} \approx$ 0 , then $F \approx F_{A} F_{B}$, which means the cascaded two converters are fully decoupled, and the impedance interaction can be neglected, then the system stability only relies on the individual well designing of the subconverters.

The minor loop gain $T_{m}$ has to be computed in a certain way for the stability assessment in voltage mode 


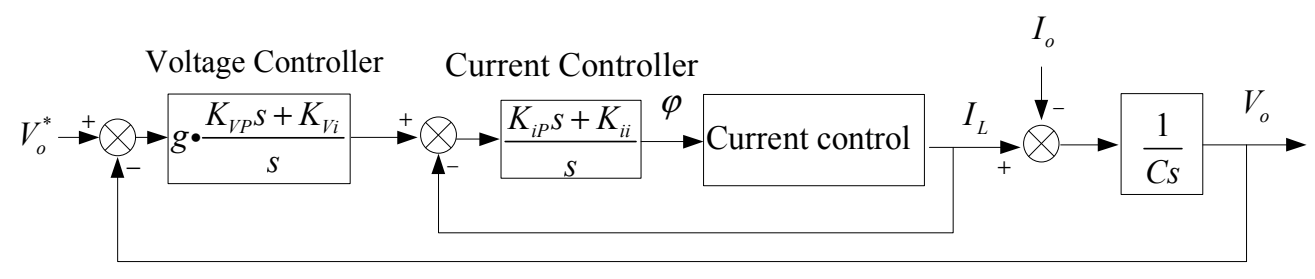

Fig. 3 Control diagram of DAB with voltage control

or current mode applications [16,17]. If sub-converter A is a voltage source, and sub-converter B is a current sink, then the impedance ratio can be expressed as:

$$
T_{m}=\frac{Z_{A o}}{Z_{B i n}}
$$

where $Z_{A o}$ is the output impedance of sub-converter A; $Z_{B i n}$ is the input impedance of sub-converter B;

If sub-converter $A$ is a current source, and subconverter $\mathrm{B}$ is a voltage sink, then the impedance ratio becomes:

$$
T_{m}=\frac{Z_{B i n}}{Z_{A o}}
$$

So the numerator of the impedance minor loop gain has to be the impedance of the subsystem containing the voltage source or sink, and the denominator has to be the impedance of the subsystem containing the current sink or source, respectively.

\section{IMPEDANCE INTERACTION MODELLING AND ANALYSIS}

The input impedance is the equivalent impedance viewed from the input source [18], and output impedance is the impedance exhibited by its output terminals to an alternating current of a particular frequency [19]. The output impedance $Z_{o}$ and input impedance $Z_{\text {in }}$ can be obtained by the small signal modeling around the operating point [20].

$$
\begin{gathered}
Z_{o}=-\frac{\Delta V_{o}}{\Delta I_{o}} \\
Z_{i n}=\frac{\Delta V_{i n}}{\Delta I_{i n}}
\end{gathered}
$$

where $\Delta V_{o}, \Delta I_{o}$ are respectively the small signals of output voltage and output current, $\Delta V_{\text {in }} \Delta I_{\text {in }}$ are the small signals of input voltage and current.

\section{A. DAB impedance under voltage control}

The power output of DAB can be controlled by the phase shift between the two bridges [21]. The average value of output current over each cycle is:

$$
I=\frac{K V_{1}}{2 \pi^{2} L_{1} f} \varphi \cdot(\pi-|\varphi|)
$$

where $V_{l}$ is the DAB input voltage, $K$ is the turns ratio of the high frequency transformer in $\mathrm{DAB}, L_{l}$ is the transformer leakage inductance, $\varphi$ is the phase shift between the $\mathrm{H}$ bridges in DAB converter.
Fig. 3 is the control diagram of DAB. The inner loop is current loop, and outer loop is voltage loop. The current and voltage variables can be found in Fig. 1.

The output voltage can be expressed as:

$$
\begin{aligned}
V_{o}= & \left.\frac{\left(K_{V P} s+K_{V i}\right) G_{D A B-I}}{C s^{2}+} V^{*} K_{V P}+K_{V i}\right) G_{D A B-I} \\
& -\frac{s}{C s^{2}+\left(K_{V P} s+K_{V i}\right) G_{D A B-I}} I_{o}
\end{aligned}
$$

where $\mathrm{K}_{\mathrm{VP}} \mathrm{K}_{\mathrm{Vi}}$ are respectively the proportional and integral parameter of the voltage controller. $C$ is the filter capacitance, $G_{D A B-I}$ is the closed loop transfer function of current loop, which can be obtained by Fig. 3 .

Then DAB output impedance can be expressed as:

$$
Z_{D A B o}=\frac{s}{C s^{2}+\left(K_{V P} s+K_{V i}\right) G_{D A B-I}}
$$

where $G_{D A B-I}$ is the current loop transfer function.

As shown in Fig. 3, in the voltage controller, $g$ is the gain of this controller, which affects the DAB output impedance. The bode plots of DAB output impedance can be shown as Fig. 4 .

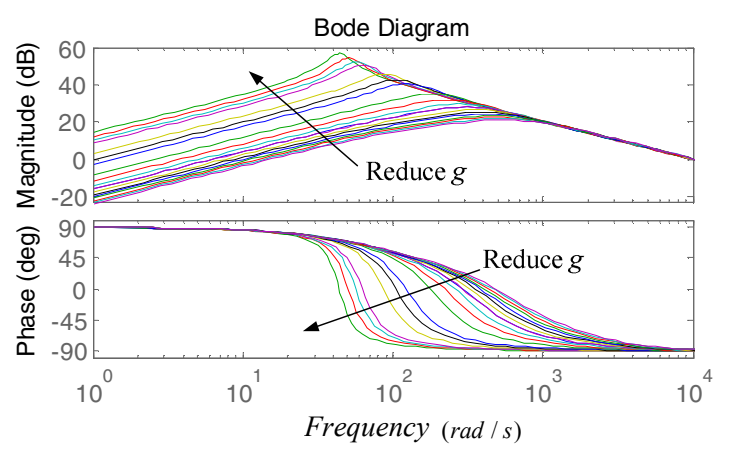

Fig. 4 Bode plots of DAB output impedance

As shown in Fig. 4, the output impedance is inductive in the low frequency range, and capacitive in the high frequency range. With the decreasing of voltage controller gain, the low frequency output impedance is increasing. According to Middlebrook criterion, the increasing of output impedance will deteriorate the stability of the cascaded system.

When the DAB power flow reverses, the block diagram is also the same as Fig. 3. Previous output current $\left(I_{o}\right)$ becomes the input current, and also the reference direction of input current changes, as:

$$
\overline{I_{\text {in }}}=-I_{o}
$$


where $\overline{I_{l n}}$ is the DAB input current in the reversed power flow.

The input voltage is the same as the output voltage in forward power flow, and then the input impedance in reversed power flow is:

$$
\overline{Z_{D A B i n}}=\frac{\Delta V_{\text {in }}}{\Delta I_{\text {in }}}=-\frac{\Delta V_{o}}{\Delta I_{o}}=Z_{D A B o}
$$

So under voltage control, DAB output impedance in forward power flow equals the input impedance in reversed power flow.

\section{$B$. Inverter impedance under constant power control}

Fig. 5 shows the topology of the inverter with the connection to grid. The inverter is under constant power control in DQ rotation frame coordinates.

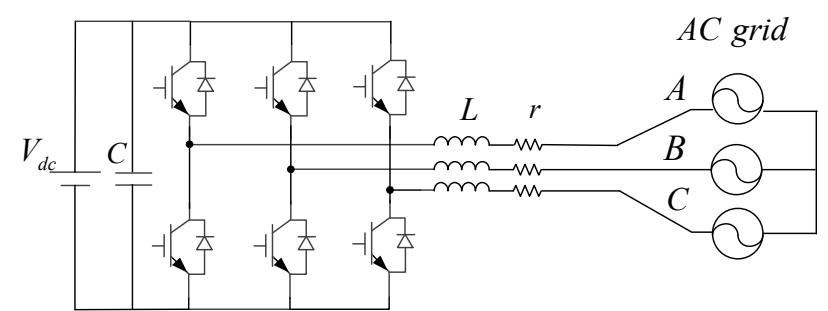

(a)Topology of the grid connected inverter

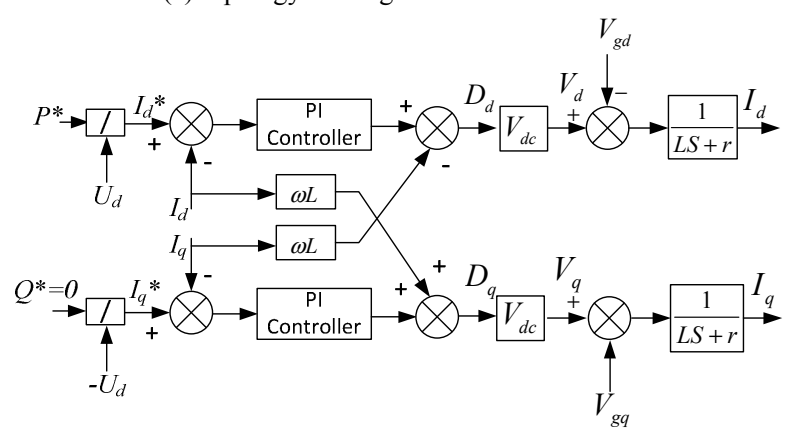

(b)Block diagram

Fig. 5 Topology and block diagram of the grid connected inverter

The inverter state equations are given as:

$$
\begin{aligned}
& L \frac{d i_{d}}{d t}=D_{d} V_{d c}-V_{d}+w L I_{q} \\
& L \frac{d i_{q}}{d t}=D_{q} V_{d c}-V_{q}-w L I_{d}
\end{aligned}
$$

where $L$ is the filter inductance and $V_{d c}$ is the DC side voltage. $w=2 \pi f, f$ is the frequency of the $\mathrm{AC}$ voltage. $V_{d}$ and $V_{q}$ are respectively the voltage on the D and $\mathrm{Q}$ axis on the rotation frame. By neglecting the switching losses, the power balance between $\mathrm{AC}$ side and DC side can be expressed as:

$$
P=\frac{3}{2}\left(V_{d} I_{d}+V_{q} I_{q}\right)=V_{d c} I_{i n}
$$

The phase lock loop (PLL) is oriented on the grid side voltage, so under steady state $V_{q}=0$; reactive power is 0 , so $I_{q}=0$. Assuming the grid voltage is constant, so the small signal $\Delta V_{d}=0$. Then the small signal of the power balance is:

$$
\frac{3}{2} U_{d} \Delta I_{d}=V_{d c} \Delta I_{i n}+\Delta V_{d c} I_{i n}
$$

where $\Delta I_{d}$ is the small signal of $\mathrm{D}$ axis current, and it can be expressed by the DC-link voltage $\Delta V_{d c}$ :

$$
\Delta I_{d}=G_{V_{d c} I_{d}}(\mathrm{~s}) \Delta V_{d c}
$$

where $G_{V_{d c} I_{d}}(s)$ is the transfer function with the input of the DC-link voltage and output of D axis current. $G_{V_{D C} I_{d}}$ can be obtained by (11), and its block diagram as shown in Fig. 6.

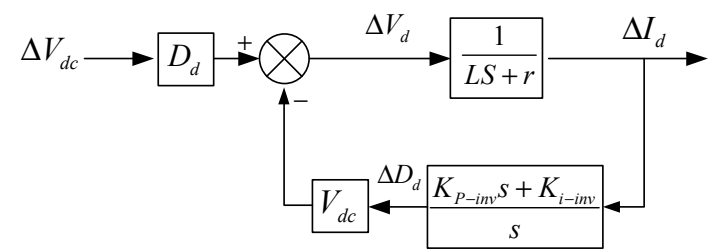

Fig. 6 Block diagram of $\boldsymbol{G}_{V_{d c} I_{d}}(\boldsymbol{s})$

In Fig. $6, D_{d}$ is the $\mathrm{D}$ axis duty ratio around the operation point, and $K_{P-i n v} K_{I-i n v}$ are respectively the proportional and integral parameters in the current controller, then $G_{V_{d c^{I}} d}(s)$ can be expressed as:

$$
G_{V_{d c} I_{d}}(s)=\frac{D_{d} s}{L s^{2}+\left(r+V_{D C} K_{P-i n v}\right) s+V_{d c} K_{i-i n v}}
$$

Based on $(13(14)(15)(16)$, the inverter input impedance can be expressed as:

$$
Z_{I N V-i n}=\frac{\Delta V_{d c}}{\Delta I_{i n}}=\frac{V_{d c}}{1.5 U_{d} G_{V_{d c} I_{d}}(s)-I_{i n}}
$$

where $I_{i n}$ is the reference input current, which can be obtained by the power reference and input voltage reference. Fig. 7 shows the bode plots of inverter input impedance. As shown in Fig. 7, the input impedance is negative in the low frequency range, with the phase shift of $-180^{\circ}$. When power flow is reversed, the inverter works in the rectifier mode, and previous input DC current becomes the output current, and the reference direction also changes. The power balance between DC side and AC side still exist as (18).

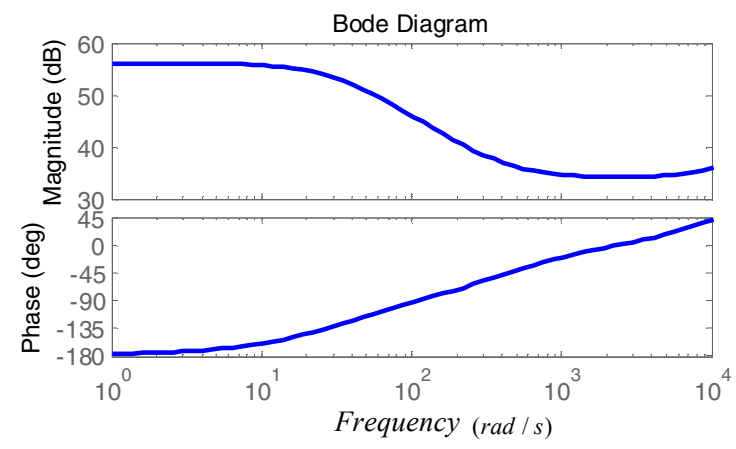

Fig. 7 Bode plots of Inverter input impedance 


$$
\frac{3}{2} U_{d} \Delta I_{d}=V_{d c} \Delta I_{o}+\Delta V_{d c} I_{o}
$$

The reference direction of DQ current also changes. Then rectifier output impedance $\overline{Z_{I N V-o}}$ can be obtained by:

$$
\overline{Z_{I N V-o}}=-\frac{\Delta V_{d c}}{\Delta I_{o}}=-\frac{V_{D C}}{1.5 \mathrm{U}_{d} \overline{G_{V_{d c} I_{d}}}(s)-I_{o}}
$$

where $\overline{G_{V_{D C I}}}(s)$ is the transfer function with the input of DC-link voltage and the output of D axis current in reversed power flow and its block diagram of $\overline{G_{V_{d c} I_{d}}}$ is shown as:

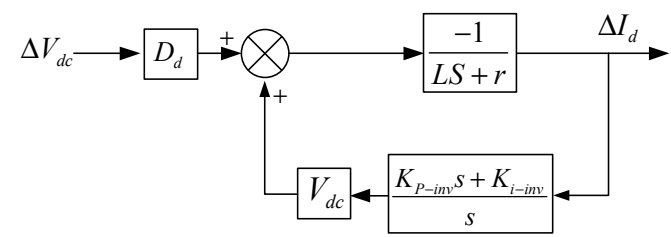

Fig. 8 Small signal block diagram from DC-link voltage to D axis current

The transfer function of $\overline{G_{V_{d c l d}}}(\mathrm{~s})$ is:

$$
\overline{G_{V_{d c} l_{d}}}(s)=\frac{-D_{d} s}{L s^{2}+\left(r+V_{d c} K_{P-i n v}\right) s+V_{d c} K_{i-i n v}}
$$

Then inverter output impedance in rectifier mode is shown in Fig. 9. As shown in Fig. 9, compared with input impedance in forward power flow, for the constant power converter with the same value of transmitted power, the output impedance in reversed power flow has an opposite sign with the input impedance in forward power flow.

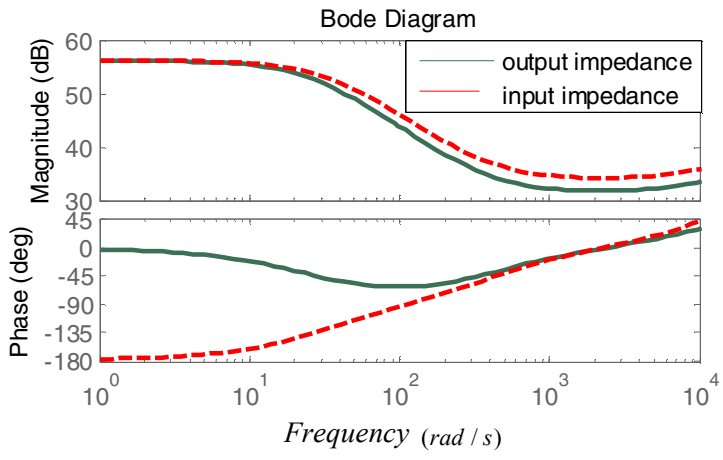

Fig. 9Bode plots of inverter output impedance in rectifier mode

\section{BIDIRECTIONAL IMPEDANCE ANALYSIS}

Based on previous modelling, the minor loop gain in the forward and reversed power flow can be obtained.

\section{A. Bode plots of the bidirectional impedance interaction}

In the forward power flow, DAB is the voltage source converter and inverter is the current load converter. Based on (2), the minor loop $T_{m}$ is:

$$
T_{m}=\frac{Z_{D A B O}}{Z_{I N V-i n}}
$$

When the power flow is reversed, DAB becomes the load converter, and inverter turns to the source converter, then based on (3), the impedance ratio $\overline{T_{m}}$ is:

$$
\overline{T_{m}}=\frac{\overline{Z_{D A B i n}}}{\overline{Z_{I N V-o}}}
$$

The bode plots of impedance minor loop gain in forward power flow $\left(T_{m}\right)$ and reversed power flow $\left(\overline{T_{m}}\right)$ can be shown in Fig. 10.

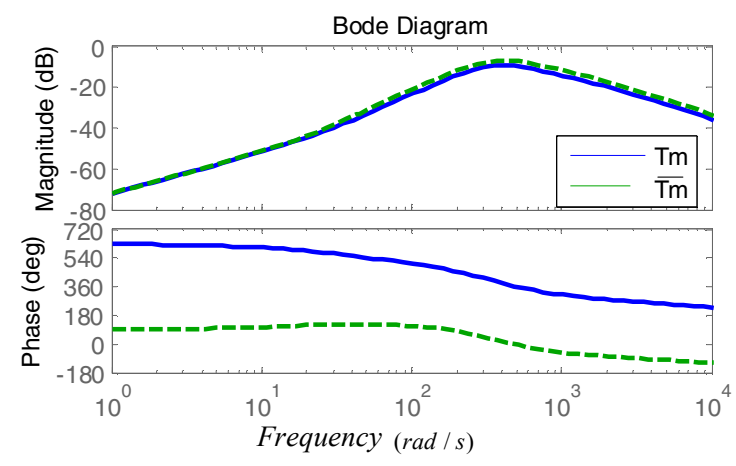

Fig. 10 Bode plots of the impedance minor loop gain $\left(\boldsymbol{T}_{\boldsymbol{m}}\right)$

As shown in Fig. 10, the phase shift of $T_{m}$ is $630^{\circ}$ ($90^{\circ}$ ) in the low frequency range. Because the inverter operates in constant power mode, and it behaves as negative impedance with the phase of $-180^{\circ}$ in the low frequency range, as in Fig. 9; the $\mathrm{DAB}$ operates in voltage control, and the output impedance has $90^{\circ}$ phase shift at low frequency range, as shown in Fig. 4 , so the total phase shift of the impedance interaction is $270^{\circ}$. In the high frequency range, the phase of DAB output impedance is $-90^{\circ}$, and the inverter input impedance is $90^{\circ}$, so in the high frequency range, the phase of impedance interaction is $-180^{\circ}$. So with the increasing of frequency, the phase of the impedance interaction shift from $630^{\circ}\left(270^{\circ}\right)$ to $180^{\circ}\left(-180^{\circ}\right.$, and this dramatic phase variation may make the system unstable, so the negative input impedance in cascaded power system needs to be taken care of [12].

And also in Fig. 10, in reversed power flow, the impedance minor loop gain $\left(\overline{T_{m}}\right)$ behaves with $90^{\circ}$ phase shift in the low frequency range, and the gain decreases with the frequency. Because, as in Fig. 4 and Fig. 9, DAB input impedance $\left(\overline{Z_{D A B I n}}\right)$ is inductive in the low frequency range, and inverter output impedance $\left(\overline{Z_{I N V-o}}\right)$ is almost resistive in the rectifier mode, so they contribute the $90^{\circ}$ phase impedance interaction the low frequency range. In the high frequency range, the $\mathrm{DAB}$ input impedance $\left(\overline{Z_{D A B I n}}\right)$ is capacitive, and inverter output impedance $\left(\bar{Z}_{I N V-o}\right)$ is mixed with resistive and capacitive, so the phase of impedance interaction is increasing from $-135^{\circ}$ to $135^{\circ}$. The phase variation of impedance minor loop gain in reversed power flow is much smaller than that in the forward power flow. 


\section{B. Nyquist plots of the bidirectional impedance interaction}

Fig. 11 shows the demonstration of the phase margin and gain margin in the nyquist plot [22].

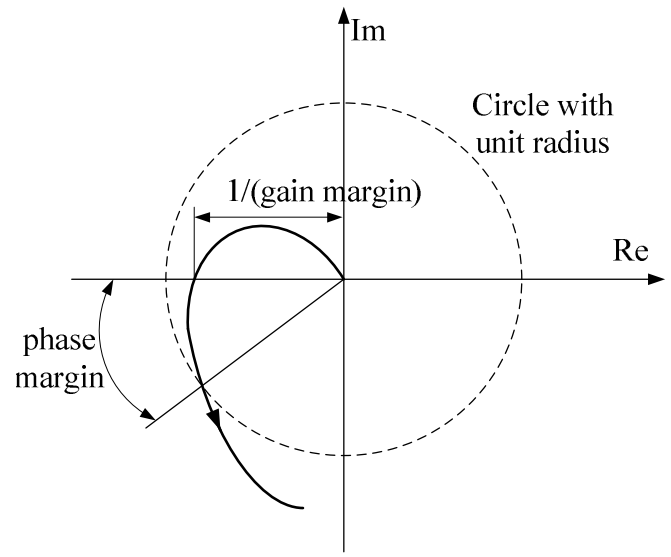

Fig. 11 Phase margin and gain margin definitions in Nyquist plot

The phase margin is the phase angle with the $-180^{\circ}$ line when the nyquist line crosses the unit circle. The gain margin is the reciprocal value of the distance, from where the nyquist line crosses the line of $-180^{\circ}$ to the origin point.

The nyquist plot of impedance minor loop gain in bidirectional power flow can be obtained by (21) and (22). Fig. 12 (a) is the nyquist plot of impedance minor loop gain in the forward power flow. In Fig. 12, the gain of DAB voltage controller decreases from $1 \mathrm{pu}$ to $0.03 \mathrm{pu}$, which means the output impedance of $\mathrm{DAB}$ are increasing, as previously depicted in Fig. 4 .

The increasing of DAB output power impedance will introduce more unstable factor in the impedance interaction. As shown in Fig. 12 (a), in forward power flow, $1 /_{G M}$ increases with the increasing of DAB output impedance, meaning that the gain margin of the minor loop gain $T_{m}$ decreases. With the increasing of DAB output impedance, the nyquist line are approaching the point of $(-1,0)$, meaning that the system becomes unstable.

Fig. 12 (b) shows the nyquist plot of impedance minor loop gain in the reversed power flow. As shown in Fig. 12 (b), $1 /_{G M} \approx 0$, so the gain margin $G M$ is much larger than that in the forward power flow. In Fig. 12 (b) when increasing the DAB impedance, there is no significant variation in the gain margin, which is more stable than that the forward in Fig. 12 (a). The nyquist line also stay away from the point of $(-1,0)$.

So without changing control methods, the cascaded converter system is more stable in reversed power flow than in the forward power flow, and it means that with the source converter under current control and load converter under voltage control, system stability will be improved, compared with source converter with voltage control and load converter with current control. Because the negative impedance in the constant power load will

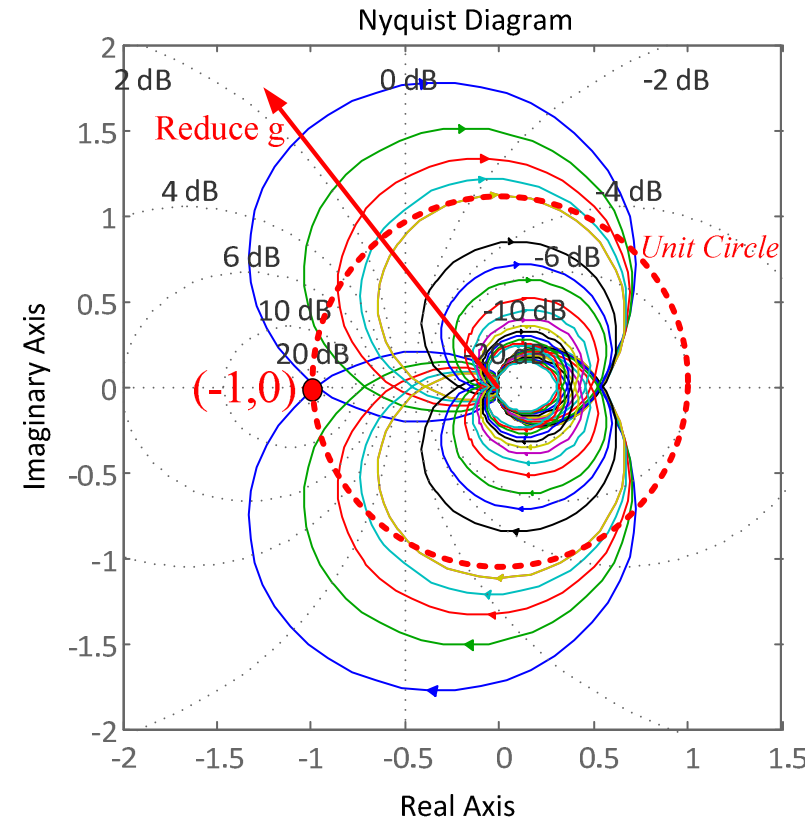

(a) Nyquist plot of the minor loop gain with the variation of voltage controller gain

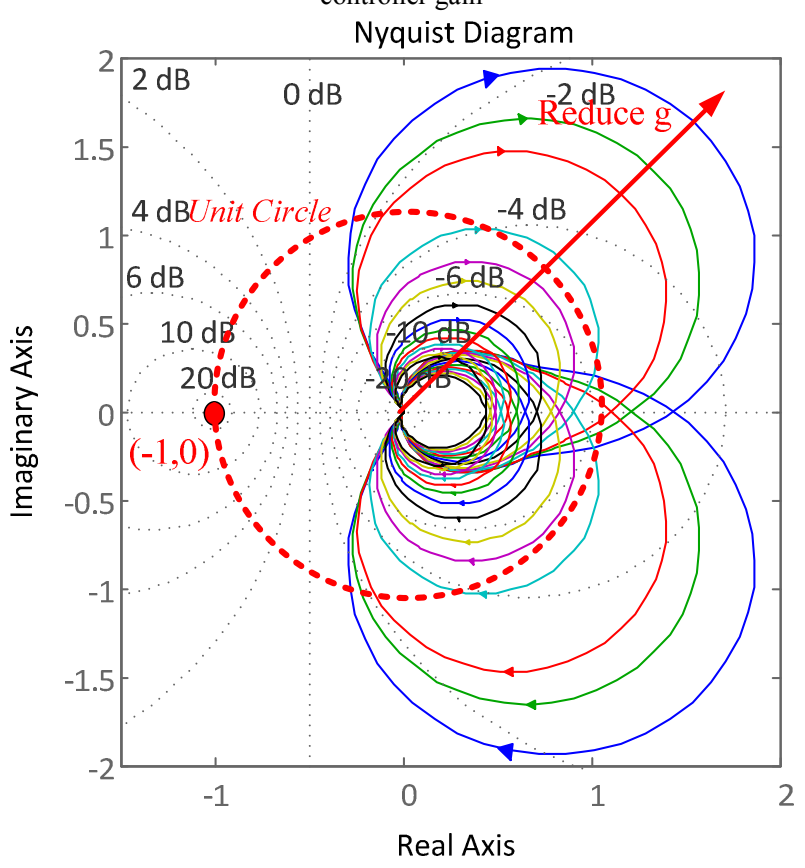

(b) Nyquist plot of the minor loop gain with the variation of voltage controller gain

Fig. 12 Nyquist plots of the minor loop gain in forward power flow

turns to resistive impedance in constant power source and it behaves more friendly in the impedance interaction.

\section{SiMULATION ANd EXPERIMENT RESULTS}

\section{A. Simulations}

In order to verify the conclusions, a model has been built under the simulation software of PLECS. The control scheme is shown in Fig. 13.

The simulation parameter is shown in Table 1. 


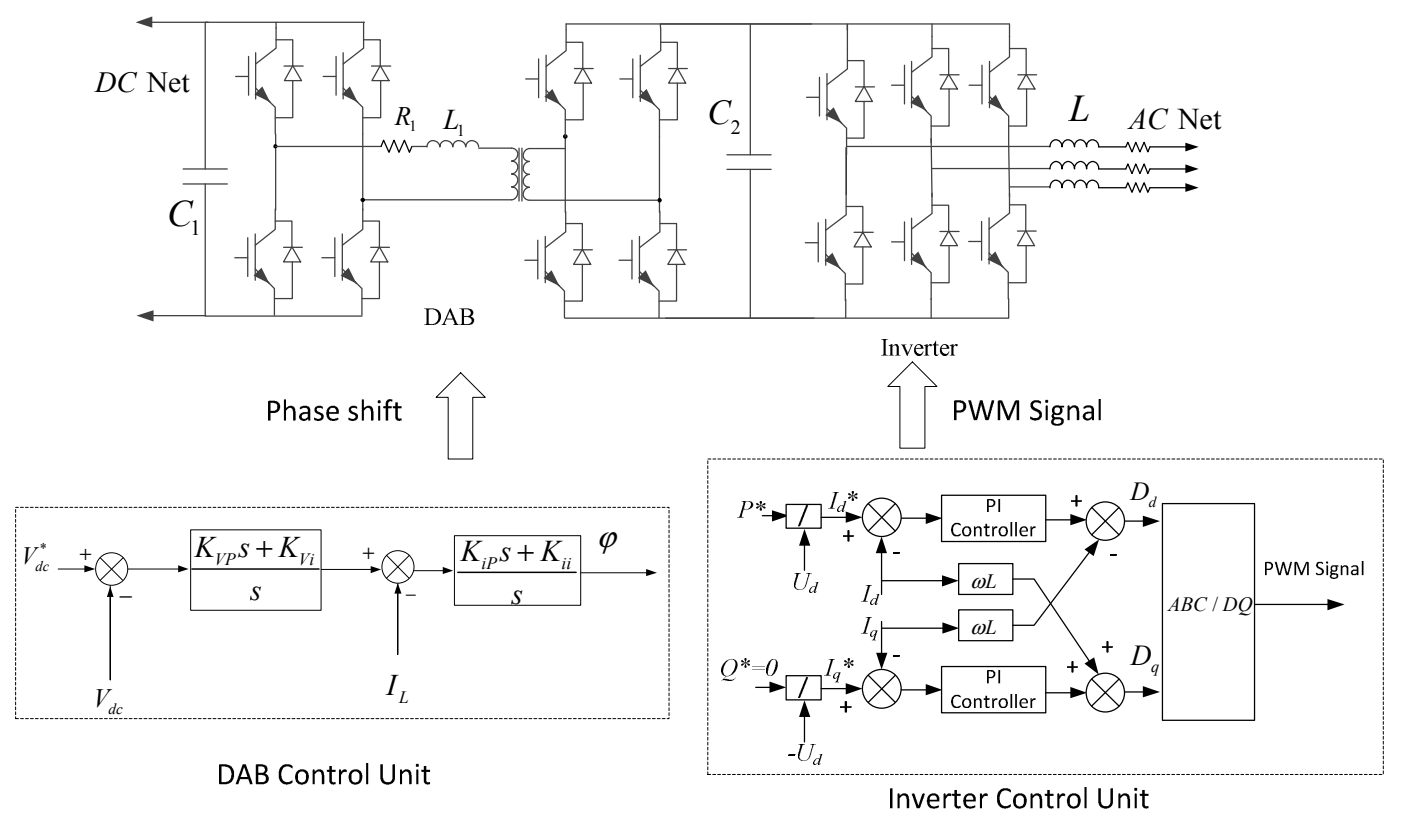

Fig. 13 Simulation model of the block diagram

TABLE I

SIMULATION PARAMETERS

\begin{tabular}{|c|c|c|c|}
\hline PARAMETER & Value & PARAMETER & VALUE \\
\hline$V_{1}$ & $400 \mathrm{~V}$ & $L$ & $3 \mathrm{mH}$ \\
\hline$V_{2}$ & $800 \mathrm{~V}$ & $C$ & $100 \mathrm{uF}$ \\
\hline$V_{a c}$ & $311 \mathrm{~V}$ & $L_{1}$ & $0.1 \mathrm{mH}$ \\
\hline
\end{tabular}

The input and output impedance can be measured by the variations of current and voltage output under certain frequency.

The measured DAB output impedance in Fig. 14. Fig. 15 shows the inverter input impedance in forward power flow and the output impedance in reversed power flow.

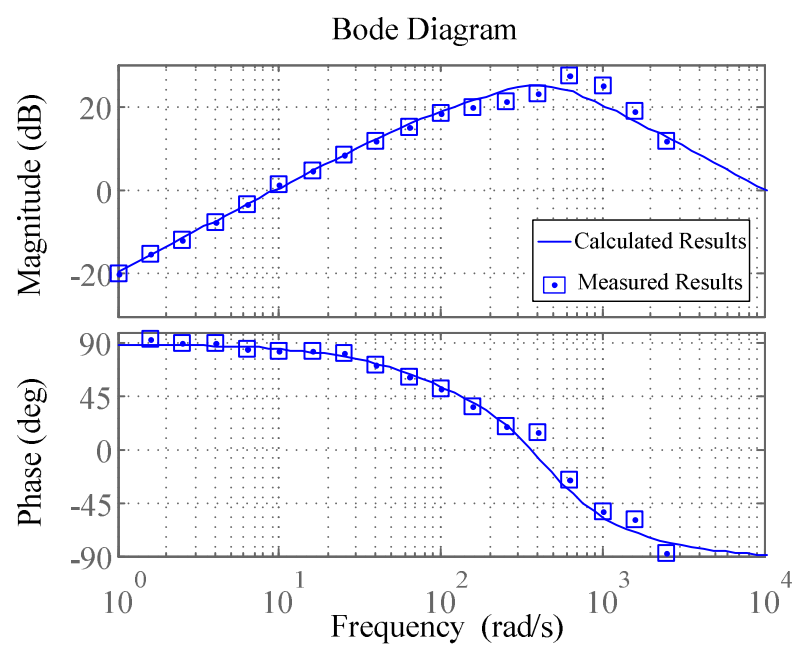

Fig. 14 DAB impedance measurement

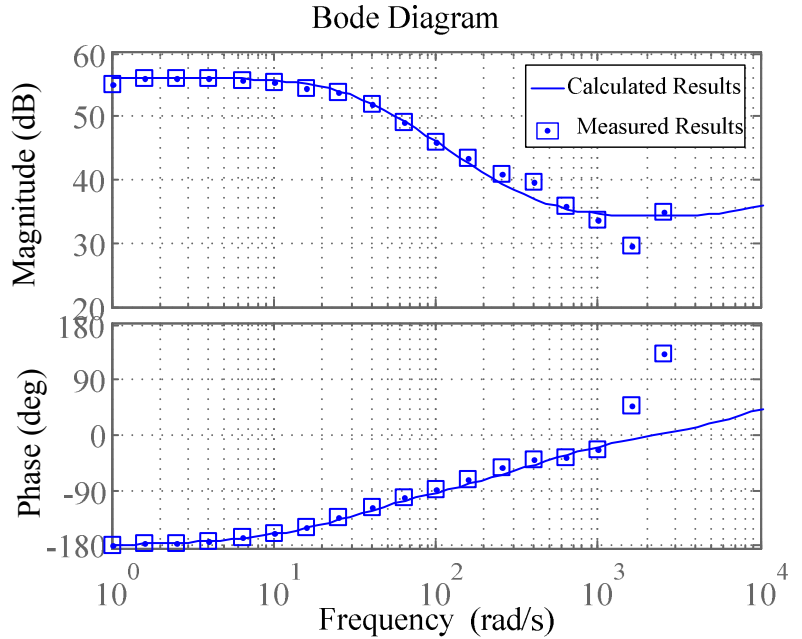

(a)Input impedance in forward power flow $(1 \mathrm{~kW})$

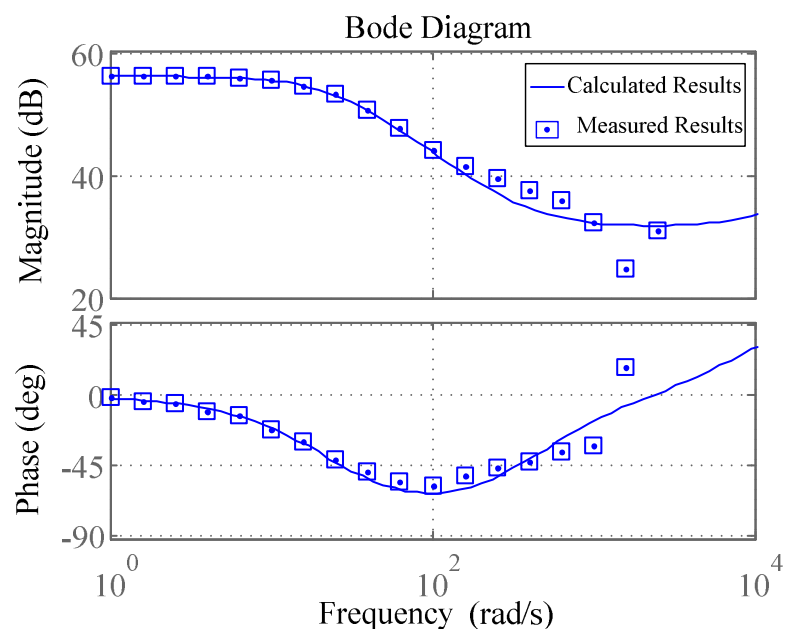

(b)Output impedance in reversed power flow $(-1 \mathrm{~kW})$ Fig. 15 Inverter bidirectional impedance measurement 
As shown in Fig. 14, the measured DAB impedance matches the calculated results well, so the DAB output impedance modelling can be verified. Fig. 15 verifies the correctness of the inverter impedance modelling.

In order to test the stability difference, DAB output impedance has been increased. The effect of the output impedance increasing on the stability performance can be shown in Fig. 12.

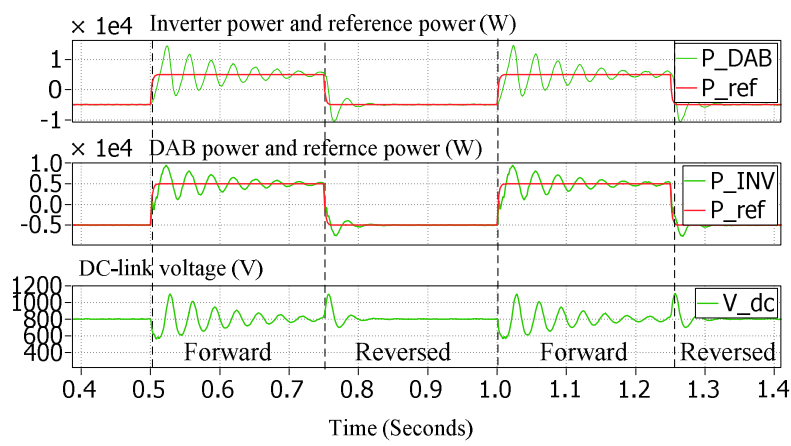

Fig. 16 Simulation wave forms of conventional control without mode switching

Fig. 16 is the simulation results, where the power reference is a time varying signal, changing between $5 \mathrm{~kW}$ and $-5 \mathrm{~kW}$, with the period of $0.5 \mathrm{~s}$. As shown, in reversed power flow $(-5 \mathrm{~kW})$, the power output and DC-link voltage are stable; in forward power flow $(5 \mathrm{~kW})$ the power output of $\mathrm{DAB}$ and inverter have serious oscillation, which means the stability in forward power flow is worse. This can verify the conclusion obtained by Fig. 12.

\section{B. Experiments results}

A scaled down prototype has also been built to test the effectiveness of the proposed control and the control unit is dSPACE1006. The experimental parameters are shown in Table 2.

The experiment scenario is that the power reference has the step changes, from $-400 \mathrm{~W}$ to $400 \mathrm{~W}$, with the period of 2 seconds, and also DAB output impedance has been increased.

As shown in Fig. 17, when power output rising from $-400 \mathrm{~W}$ to $400 \mathrm{~W}$, there are significant oscillations on the power output of DAB and inverter, as well as the DC-link voltage. When power output decreases from $400 \mathrm{~W}$ to $400 \mathrm{~W}$, the oscillations become smaller on both the sub-

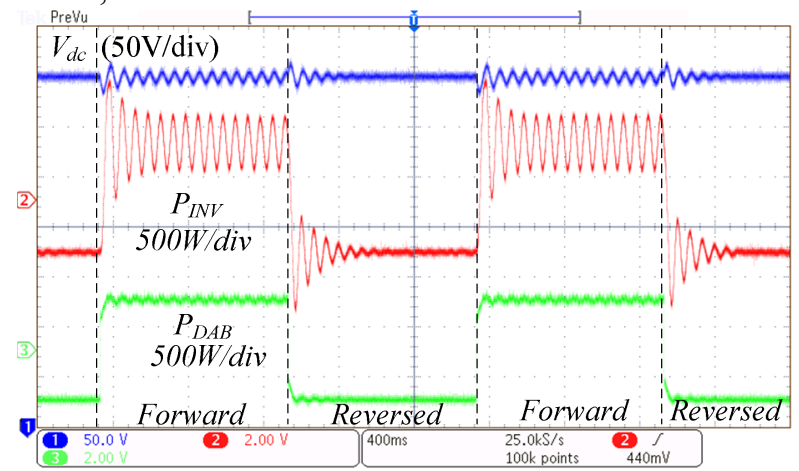

Fig. 17 Experimental results of the sub converter power output and the DC-link voltage

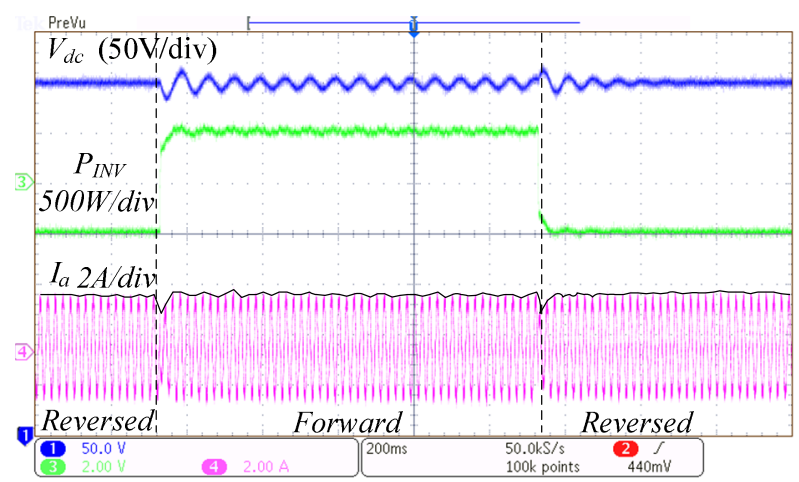

(a)Experimental results of DC-link voltage, inverter power and current output

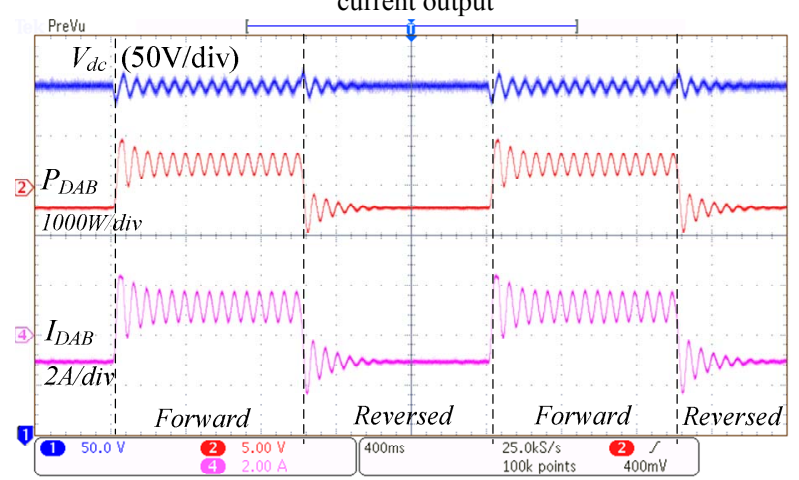

(b)Experimental results of DC-link voltage, DAB power and current output

Fig. 18 Experimental wave forms with power reference from $-400 \mathrm{~W}$ to 400W

converter power output and the DC-link voltage, and system become stable sooner. So in reversed power flow, the cascaded system is more stable than the forward power flow.

Fig. 18 shows the current wave forms of inverter and DAB when the power flow changing between $400 \mathrm{~W}$ and $-400 \mathrm{~W}$. As shown in Fig. 18(a), inverter output current has smaller oscillation in reversed power flow $(-400 \mathrm{~W})$ than that in the forward power flow (400W). In Fig. 18 (b), when the power output is $400 \mathrm{~W}$, the oscillation in the DAB power output and current output are significant. In the reversed power flow $(-400 \mathrm{~W})$, the relevant oscillations are reduced and system become stable soon.

\section{CONCLUSION}

This paper analyzed the bidirectional impedance interaction for the two stages cascaded converter, and shows that the variation of power flow will change the impedance interaction, because the CPLs converter behaves the negative impedance as the load and resistive impedance as the source. The DAB impedance is obtained from the control block diagram, and the inverter impedance is calculated through the small signal model. The impedance interaction in both forward power flow and reversed power flow is offered. Both the bode plots and nyquist plots are offered to show the performance of the bidirectional impedance interaction. The result shows that with $\mathrm{DAB}$ converter controlling the $\mathrm{DC}$-link voltage 
and inverter controlling the power output, the system is more stable when power flow from inverter to the $\mathrm{DAB}$, compared with power from DAB to inverter. This means that in cascaded converter system, if source converter control the power output and load converter control the DC-link voltage, the system stability will be improved. The results have been validated by both the simulation and experimental results.

\section{APPENDIX}

TABLE II

SiMULATION PARAMETERS

\begin{tabular}{|c|c||c|c|}
\hline $\begin{array}{c}\text { DAB } \\
\text { Parameter }\end{array}$ & Value & $\begin{array}{c}\text { Inverter } \\
\text { Parameter }\end{array}$ & Value \\
\hline$($ VI) & $125 \mathrm{~V}$ & AC voltage & $150 \mathrm{~V}$ \\
\hline$(V 2)$ & $400 \mathrm{~V}$ & $\begin{array}{c}\text { Inductor of } \mathrm{L} \\
\text { filter }\end{array}$ & $3 \mathrm{mH}$ \\
\hline$K$ & $1: 3.2$ & $\begin{array}{c}\text { Switching } \\
\text { Frequency }\end{array}$ & $10 \mathrm{kHz}$ \\
\hline$L_{l}$ & $200 \mathrm{uH}$ & & \\
\hline$C$ & $300 \mathrm{uF}$ & & \\
\hline$f$ & $10 \mathrm{k} \mathrm{Hz}$ & & \\
\hline
\end{tabular}

\section{ACKNOWLEDGEMENTS}

The authors would like to thank the Danish Council for Strategic Research for providing the financial support under the project "Development of a Secure, Economic and Environmentally-friendly Modern Power Systems" (DSF 09-067255.

\section{REFERENCES}

[1] Xinyun Liu, Andrew J. Forsyth, Andrew M. Cross, "Negative input-resistance compensator for a constant power load," IEEE Transactions on Industrial Electronics, Vol. 54, No. 6, December 2007.

[2] WayneW.Weaver, PhilipT. Krein , "Optimal geometric control of power buffers," IEEE Transactions on Power Electronics, vol.24, no. 5, May 2009.

[3] Xiaofang Feng, Jinjun Liu, Fred C. Lee, "Impedance specifications for stable DC distributed power system," IEEE Transactions on Power Electronics, vol.17 no. 2, March 2002.

[4] D. Dong, F. Luo, X. Zhang, D. Boroyevich, P. Mattavelli, "Grid-Interface bidirectional converter for residential DC distribution systems-part 1: High-Density two-stage topology," IEEE Transactions on Power Electronics, vol. 28, no. 4, April 2013

[5] M. Jiang, V. G. Agelidis, "A minimum power-processingstage fuel-cell energy system based on a boost-inverter with a bidirectional Backup Battery Storage", IEEE Transactions on Power Electronics, vol. 26, nO. 5. MAY 2011.

[6] Leandro Roggia, Luciano Schuch, Jos'e Eduardo Baggio, Cassiano Rech, Jos'e Renes Pinheiro, "Integrated fullbridge-forward DC-DC converter for a residential microgrid application," IEEE Transactions on Power Electronics, vol. 28, nO. 4, April 2013.

[7] R.D. Middlebrook, "Input filter considerations in design and application of switching regulators, " in Proc. IEEE IAS, 1979, pp 366-382.

[8] Fred. C. LEE, Yuan Yu, "Input-filter design for switching regulators," in Proc. NAECON, 1979, pp: 627-634.
[9] Jiabin Wang, David Howe, "A power shaping stabilizing control strategy for DC power systems with constant power loads," IEEE Transactions on Power Electronics, vol. 23 , no. 6 , November.

[10] Harish Krishnamurthy, Raja Ayyanar, "Stability Analysis of Cascaded Converter for Bidirectional Power Flow Applications", INTELEC 2008. IEEE 30th International 2008, Page(s): $1-8$.

[11] Honglin Zhou, Shuai Xiao, Geng Yang, Hua Geng, "Modeling and control for a bidirectional buck-boost cascade inverter," IEEE Transaction on Power Electronics, vol. 27, no. 3, March, 2012.

[12] Min Chen, Jian Sun, "Low-Frequency input impedance modelling of boost single-phase PFC converters," IEEE Transactions on Power Electronics, vol. 22, nO. 4, July. 2007.

[13] Carl M. Wildrick, Fred C. Lee, Bo H. Cho, Byungcho Choi, "A method of defining the load impedance specification for a stable distributed power system," IEEE Transactions on Power Electronics, vol. 10, no. 3. May, 1995.

[14] Shousong Hu, "Automatic control theory," [M] Science Press, 2001.

[15] S. Vest, T. Suntio, J. A. Oliver, R. Prieto, J. A. Cobos, "Impedance-based stability and transient-performance assessment applying maximum peak criteria," IEEE Transactions on Power Electronics, vol. 28, no. 5, May. 2013

[16] J. Lepp“aaho, J. Huusari, L. Nousiainen, J. Puukko, and T. Suntio, "Dynamic properties and stability assessment of current-fed converters in photovoltaic applications," IEEJ Trans. Ind. Appl., vol. 131, no. 8, pp. 976-984, Aug. 2011

[17] J. Sun, "Impedance-based stability criterion for gridconnected inverters," IEEE Trans. Power Electron., vol. 26, no. 11, pp. 3075-3078, Nov. 2011.

[18] From the website of Wiki, input impedance, http://en.wikipedia.org/wiki/Input_impedance

[19] From the website of Wiki, output impedance, http://en.wikipedia.org/wiki/Output_impedance

[20] Yuri Panov, Milan Jovanovic, "Practical issues of input/output impedance measurements in swithching power supplies and application of measured data to stability analysis," Applied Power Electronics Conference and Expositions, 2005, Austin, TX, pp. 1339 - 1345, vol. 2.

[21] Yangguang Yan, "Bidirectional DC converter," [M] Jiangsu Science and Technology Press, 2004.

[22] A. Emadi, A. Khaligh, C. H. Rivetta, and G. A. Williamson, "Constant power loads and negative impedance instability in automotive systems: Definition, modeling, stability, and control of power electronic converters and motor drives," IEEE Trans. Veh. Technol., vol. 55, no. 4, pp. 1112-1125, Jul. 2006. 